\title{
DIEZ REFLEXIONES SOBRE MEDIACIÓN PEDAGÓGICA
}

\author{
TEN REFLECTIONS ON PEDAGOGICAL MEDIATION
}

DEZ REFLEXÕES SOBRE A MEDIAÇÃO PEDAGÓGICA

\section{Marcelo Humberto Godoy Gálvez ${ }^{1}$, Johanna Cortes Veliz ${ }^{2}$, Rodrigo Espinoza Vásquez ${ }^{3}$, Mónica Rengifo Oyarce ${ }^{4}$}

${ }^{1}$ Universidad Santo Tomás, La Serena, Chile, marcelo.godoy@santotomas.cl

${ }^{2}$ Fundación Integra, Chile, jcortes@integra.cl

${ }^{3}$ Escuela Elqui Libre, Chile, respinoza.psicopedagogia@gmail.com

${ }^{4}$ Universidad de La Serena, Chile, mrengifo@userena.cl

Fecha de recepción: 14 de enero de 2021

Fecha de aceptación: 27 de febrero de 2021

\section{RESUMEN}

El presente texto es una reflexión que surge de la revisión bibliográfica y de experiencias educativas para la comprensión del fenómeno de la mediación pedagógica. Acercamos nuestros pensamientos hacia la idea de mediación como un entramado de experiencias educativas, donde se produce la creación de relaciones posibles entre quienes viven la situación de aprendizaje.

Esta reflexión comprende la mediación a partir de la ética y fundadas en el amor y la aceptación. Asimismo, concebimos el aprendizaje como una transformación profunda del ser, pues aprender es sinónimo de vida.

Palabras Claves: mediación; aprendizaje; ética; transformación; vida.

\section{ABSTRACT}

The following article comes out of the reflection and bibliographic revision of educational experiences in order to understand the educational mediation phenomenon. Our approach to mediation is as the weaving of educational experiences where relationships are built among those in search of a learning situation.

This reflection understands mediation based on ethics and founded in love and acceptance. Furthermore, we believe learning as a profound transformation of the being, since learning is a synonym of life.

Key words: mediation; learning; ethics; transformation; life.

\section{RESUMO}

O presente estudo é uma reflexão que surge de uma revisão bibliográfica e de experiências educativas desenvolvidas para a compreensão do fenômeno de mediação pedagógica. Inicialmente, entendendo a mediação como uma estrutura de experiências educativas, onde se produz a criação de relações possíveis entre os atores que vivem a situação de aprendizagem. Essa reflexão compreende a mediação a partir da ética, fundamentada no amor e na aceitação. Sendo assim, compreendendo a aprendizagem com uma transformação profunda do indivíduo, considerando que aprender é sinônimo de vida.

Palavras chaves: mediação; aprendizagem; ética; transformação; vida 


\section{1.- INTRODUCCION}

La mediación educativa implica generar las condiciones para la propensión a aprender. Tanto el aprender como el mediar, se intersectan y entraman para provocar lo que, en palabras de Calvo (2017), es la educación como creación de relaciones posibles.

La mediación se nutre de diversos elementos que tienen como corolario el respeto y valoración por l-s otrs. ${ }^{1}$ Es por ello que reflexionamos y comprendemos la mediación educativa a partir de la generación de redes que surgen en una relación de convivencia emergente, caótica, autoorganizada, que se funda en la ética y la emocionalidad del amor. Ella se vive desde la transformación emergente que ocurre en las relaciones humanas. La mediación, finalmente es un ejercicio de libertad.

En este texto desarrollamos las ideas asociadas a cómo pensamos y vivimos la mediación educativa en un aula viva. Disoñamos una praxis educativa que concibe el aula como un sistema vivo, abierto y complejo. Esta aula viva es un estar-siendo-ocurriendo que se nutre de sí misma y del entorno, un contexto de creación de relaciones posibles, donde preveer la ocurrencia de un determinado aprendizaje, es simplemente una ilusión, un espejismo (Calvo, 2017). Implicados en el entramado relacional ético, estético y político, expone el resultado de un diálogo orgánico de l-s autor-s que pretende ampliar la mirada de la mediación como un recurso exclusivamente técnico, a su estatus de proceso humano y social de carácter performativo del espacio/tiempo de convivencia general y educativo en particular. Por ello no está libre de las paradojas y contradicciones que actualmente se viven en el sistema educativo formal. La mediación, asumida como proceso vital, fluye en un espacio/tiempo que colapsa un instante o instantes autoorganizados, donde la experiencia adopta un sentido peculiar en los procesos vitales de cada un- y de tod-s.

${ }^{1}$ Hemos optado por el uso de "-" para la asignación de género, pues nos parece que representa con mayor libertad la amplitud humana, a diferencia de lo dicotómicamente establecido entre lo femenino y lo masculino.

\section{2.- PRIMERA REFLEXIÓN: LA MEDIACIÓN SE DA EN EL SENO DE UNA RED DE RELACIONES VIVAS}

El nacimiento de un bebé humano marca un acontecimiento en su trayectoria vital. Su llegada al mundo es un proceso de acoplamiento estructural con el medio, que ya no es solo su madre, sino a un nicho vital que lo envuelve y lo acoge en una dimensión histórica, social y cultural. Este espacio/tiempo es una red de interacciones, relaciones interdependientes y nutritivas con su proceso autopoiético, propio de los seres vivos que se extiende durante toda la vida.

Desde esta perspectiva entendemos la vida, como patrones de relaciones implicados (Capra,1992), distinguiendo la interdependencia, la co-evolución, las comunidades orgánicas que se renuevan creando formas adaptables y resistentes en un entretejido. En las complejidades no hay seres vivos que puedan subsistir fuera de un nicho ecológico, no hay existencia independiente, múltiples mediadores son co-partícipes de esta red de relaciones cuyos encuentros pasan por la conservación de los procesos vitales cuyo devenir está en todo su potencial, y a la vez el cambio que implica admitir que el encuentro en la mediación con el otro es siempre desde la vida misma, desde una proximidad en su existencia, en su misterio, en su potencial, cuya presencia y encuentro conmueve, siendo a la vez constitutivo y configurador.

La mediación para el aprendizaje se crea y se recrea en cada encuentro, no está predeterminada por un decálogo del buen actuar, no es un guión a seguir, ni menos un método a adoptar: está a la deriva de la propiedad dinámica de los seres vivos, lo que implica que estamos en coordinaciones permanentes con el acontecer, las necesidades, las corporalidades, las historias, los sentires, los intereses, las prioridades, los tiempos, las comprensiones, los deseos de intimidad, la inspiración del momento. No hay una pauta, pero hay un patrón, hay un sentir, hay una creencia, hay una conciencia. 
La mediación como acoplamiento estructural (Varela y Maturana, 2013), se vive como una experiencia de transformación, reestructuración y creación mutua entre mediador y persona mediada, ambos en una coordinación emocional, lingüística, sensorial, y corporal, en una posición entramada que fluye en una compleja red de relaciones de co-existencia e interdependencia que se modifican. Si hay vida, hay aprendizaje.

La mediación no se circunscribe al aula escolar, sino que la trasciende a la vida misma. Un aula viva, por lo tanto, es el nicho ecológico donde habita y convive la experiencia nutritiva del aprender humano.

\section{3.- SEGUNDA REFLEXIÓN: LA MEDIACIÓN DES- DE UNA ÉTICA DEL ENCUENTRO}

Avanzar en la comprensión de las complejidades, significa encarnar una nueva mirada, que amplía la reducida percepción en la cual hemos transitado, nos permite desdibujar y enriquecer algunos límites conceptuales, salir de la ficción de la existencia de un punto de vista, de la supremacía de un conocimiento, cuya hegemonía ha sido naturalizada y encarnada durante siglos y particularmente en nuestra trayectoria vital durante varias décadas.

Las complejidades nos permiten avanzar en comprender que la vida ocurre en una red de relaciones, cuya dinámica se da en un constante fluir de intercambios, corporalidades, situaciones y aconteceres. La trama la comprendemos desde la interconexión, la co-evolución, la simbiosis, alejada de las estructuras jerárquicas, verticales, su configuración es de una red, cuyas conexiones no poseen una centralidad, sino holones desde donde surgen nuevas conexiones que se amplifican, se curvan y se vuelven a entramar.

En la complejidad la ética que emerge se aparta de una óptica y una acústica del control, su estética deja de ser dicotómica y se amplía en una gama de posibilidades.

Comprender la mediación desde esta nueva mirada, significa necesariamente crear nuevos significados y nuevas atribuciones, por esto concebimos la mediación desde una ética del encuentro, es decir, nos sitúa en el entramado junto a otr-s.

La mediación para el aprendizaje, desde una ética del encuentro, nos invita a hacer lecturas y observaciones atentas de las señales sutiles que nos sitúan en un umbral, cuya espera no es pasiva, ni distante, sino implicada, en una escucha permanente para interpretar en qué momento es necesario el gesto, la palabra, la interacción y cruzar ese umbral con una disposición pertinente que conduce a un espacio y un tiempo irrepetibles que permiten vivir la posibilidad entramarse en el aprendizaje. La fugacidad, la constancia, la acogida, el desafío, la compañía, pueden ser las consignas cuando el aprendiz está en su propio proceso.

\section{4.- TERCERA REFLEXIÓN: LA MEDIACIÓN OCU- RRE EN LA EMOCIÓN FUNDAMENTAL QUE ES EL AMOR}

El amor es la negación de la muerte, por lo tanto, al hablar del amor como esencia de la mediación es asumir un compromiso con la vida, con una filosofía y forma de vida. No se trata de una mera declaración, implica construir y sostener una red de relaciones posibles entre quienes vivimos la mediación.

La mediación es un acto de amar, de "aceptación del otro como un legítimo otro en su diferencia" (Maturana,1990). Implica reconocer, validar, amar la propia experiencia. Y, desde ahí, con humildad, conducirnos a reconocer, validar y amar la experiencia de otr-s, en la configuración de un nosotrxs que da vida al espacio/ tiempo "espiritual, emocional y cognitivo" de un aula viva, donde la mediación germina como un "estado/ acción” de la consciencia. En este espacio entramado y complejo, emerge el proceso de aprender al cual propendemos naturalmente (Calvo, 2012).

La mediación es, para nosotr-s, más que (o junto con) una estrategia de enseñanza/aprendizaje, una filosofía 
y actitud ante la vida que genera vínculos seguros entre educador-s y aprendientes desde un espacio emocional amoroso. En este proceso, el mediador es intencional, perseverante, paciente y da sentido a las experiencias para que éstas sean trascendentes. Pero a la vez es receptivo ante la posibilidad de ser también mediado por lxs otrxs y aceptar la negativa del otrx a ser mediado. En tal sentido, tod-s propendemos a ser mediadores, vinculándonos desde un emocionar amoroso en un sentir compartido. En definitiva, en el centro está la necesidad del otr- antes que la propia (aún cuando no somos ajenos a ésta).

La mediación es un acto amoroso intencionado por parte del mediador (López de Maturana, 2017), que deviene en transformación. Tal como el pie transforma al zapato, éste transforma al pie. Es decir, la mediación aún intencionada encierra una sorpresa, lo inesperado. La mediación es convivencia humana, es vivirse una experiencia, por ello contiene en su regazo la riqueza de sentir y convivir en un tiempo y espacio de co-creación.

La mediación implica lograr una sintonía, que expresa la intimidad de cada quién. Esas experiencias se entrelazan desde la situación única que representa cada momento. Hoy cobran sentido cosas que antes no lo hacían, porque el sesgo de la realidad propia pugna por imponer su visión. Conectarse con aquello significa conectarse con, por ejemplo, una corporeidad hoy ausente dada la virtualidad de nuestras relaciones derivado del COVID-19. Soltar el hoy enfrentándonos a la experiencia que emerge es parte del aprendizaje de la vida misma en el que mediamos y somos mediados. Un momento y otro hacen sentido, pero sentido desde la experiencia única del devenir.

Desde la mediación podemos volver a ese lugar de infancia donde somos capaces de valorar y amar genuina y espontáneamente la relación que se produce con otras y otros, situados en un espacio emocional fundamentalmente amoroso.

45|Diez reflexiones sobre mediación pedagógica
Toda experiencia de la vida nos media. Un texto, un atardecer, un color, una sensación, todo evento que simplemente ocurre y nos interpela. Asimismo, uno es mediado por otr-s y por la propia experiencia en un bucle recursivo que nos interpela en nuestro presente, nuestro día a día.

\section{5.- CUARTA REFLEXIÓN: LA MEDIACIÓN COMO INFLUENCIA SUTIL Y UN BUCLE DE RETROALI- MENTACIÓN POSITIVA}

LSiempre estamos al borde de algo. Las sutilezas son las hebras más finas de una tela, pero no son evidentes, sino entramadas. En la mediación somos parte de esas hebras que tejen con paciencia la transformación que provoca el aprendizaje. Uno a uno los aprendizajes van surgiendo en ese espacio de intersubjetividad y singularidad, donde la experiencia vivida nos permite crear nuevas relaciones posibles. En ocasiones un gesto, un tono, una mirada o una palabra nos llevan a recorrer un camino impensado, que sorprende en la medida que avanza. Quienes median permanecen en el umbral, atendiendo a las condiciones que se generan y que en ocasiones son provocadas, para ingresar en el territorio del aprendizaje.

Influir (in-fluir) es una invitación a movilizar. El aprendizaje contiene movimiento en toda índole, no se detiene. La mediación, que nos invita a ser nosotr-s mism-s, es un movimiento permanente que se alimenta de las sutilezas que experimentamos nosotr-s l-s human-s.

Los sistemas complejos son sistemas abiertos que interactúan con el entorno, evolucionan y se enriquecen fluyendo entre bucles de retroalimentación positiva y negativa, lo que les permite ir creando nuevas estructuras en equilibrio dinámico (Briggs y Peat, 1999)

En la medida que nos permitimos experienciar desde el caos, es decir, soltar la predictibilidad, la certeza y el control, la estructura tiende a la autoorganización creativa, la creatividad puede aparecer en la turbulencia de una discusión, en la confusión de un pensamiento, 
emergiendo como una nueva relación que se establece, una nueva comprensión que todos podemos experimentar, la creatividad se cuece a fuego lento, en el devenir del proceso histórico de cada unx, lo podemos vivenciar al escribir estas reflexiones, nos permitimos la confusión, la incertidumbre respecto de dónde desembocarán nuestros razonamientos, estamos dispuest$\mathrm{s}$ a fluir en las relaciones que vamos estableciendo.

En la mediación para el aprendizaje, el mediador perturba para entrar en un bucle de retroalimentación positiva: propone, invita, ofrece, otorga y en un patrón de comportamiento que implica estar ni lo suficientemente cerca para dirigir o controlar, ni lo suficientemente lejos para no involucrarse, observa la respuesta coherente (entendiendo que siempre la conducta del sujeto es coherente con su nicho vital, según lo plantea Maturana) y entonces vuelve a desafiar, en un proceso constante, de manera que en la medida que otorgamos, ofrecemos y disponemos con niveles de complejidad coherentes, el sistema en algún punto se bifurca y se amplifica, en un bucle de retroalimentación positiva que permite el cambio de la estructura.

El, la, l-s sujet-s mediad-s no son meros receptor-s pasiv-s de esta experiencia de mediación, son capaces a su vez de generar un bucle de retroalimentación negativa en la que tiende a reducir la perturbación del medio y le permite conservar su identidad en tanto ser cognitivo, que es su propia implementación, su historia y su acción.

\section{6.- QUINTA REFLEXIÓN: MEDIACIÓN PARA UN APRENDIZAJE EMERGENTE, CAÓTICO Y AUTO ORGANIZADO}

El aprendizaje al igual que la vida es un proceso creativo, abierto, imprevisible, alejado del equilibrio, con amplios grados de libertad, ocurre en la corporalidad de cada ser humano. Los sistemas complejos dan cuenta de un principio dinámico que es la auto organización, la cual tiende a la autorenovación para con- servación y la autotrascendencia para la evolución y desarrollo, ambos procesos complementarios (Capra, 1992) permiten la aparición de nuevas propiedades y estructuras, creando estados cualitativamente nuevos.

Tal como el Universo surge de una explosión situada en la nada (Hawkings, 2018), que posteriormente da pie a una caotización de los elementos y luego de millones de años pueda ir formando nuevos escenarios y nuevas especies en un contexto que logra establecer un equilibrio en los márgenes del caos inicial, es como vamos comprendiendo la mediación. Un Universo humano explota en la medida que se genera el caos en su interior, pero en este caso, puede ser una sutileza la que provoque esto y se convierta en una cascada de transformaciones, quizás mínimas, veloces, lentas y/o duraderas, pero con la claridad que algo está siendoocurriendo.

La mediación pedagógica nos genera caos, porque asume la posibilidad de la transformación y la sorpresa. Tal como lo ha hecho nuestro Universo que sigue caótico en su expansión, la nuestra se sitúa en el territorio del ser, eclosiona, se abre como pétalo de flor. Nuestro ser es un aula incierta, pues en el "ser aulas" es donde nos ocurre el aprendizaje: el territorio es el cuerpo y sus experiencias. La propiedad emergente de la vida no se puede predecir, el elemento caótico se contrapone al orden estancado. Para comprender el proceso de aprendizaje es necesario pasar de lo pre-establecido, preconfigurado, ordenado y estructurado, a nuevas configuraciones. En palabras de Assman (2002) entendemos que los procesos emergentes adquieren una dinámica propia en la medida que van mostrando bifurcaciones, nuevos e infinitos caminos creativos; entonces surge la oportunidad de no estancarse, de no fosilizar los parámetros previos y permitir crear nuevas e infinitas posibilidades. La mediación pedagógica es una oda al encuentro y a la riqueza de la emergencia, que propician el aprender desde la autoorganización de la experiencia, permitiendo que emerja la diversidad, favoreciendo la fractalización de infinitas formas y ex- 
presiones del conocimiento, entendiendo este último como una interpretación que surge de nuestra capacidad de comprensión (Varela, 2016).

\section{7.- SEXTA REFLEXIÓN: LA MEDIACIÓN OCU- RRE EN UN TIEMPO FRACTAL}

El tiempo cronológico como la única dimensión vivenciada es un tiempo separado de la experiencia humana, reducido a un tiempo mecánico. El Cronos devora, no permite el gozo, ni la contemplación, es el tiempo de la productividad, la linealidad, reducido a números. Es una lucha contra el tiempo: ganar tiempo, tener tiempo, contratiempos, matar el tiempo.

La experiencia cotidiana en la cual vivimos privilegia el tiempo lineal, numérico, cuantificable, rentable, productivo. Nuestra relación con el tiempo la vivimos permanentemente desde la productividad y la esquizofrenia de pretender hacer la mayor cantidad de actividades en el menor tiempo posible, es la ficción de la optimización del tiempo. Sin embargo, en ocasiones es posible experimentar un tiempo fractal, como ahora, en este instante, al escribir, cuando nos conectamos con nuestra emocionalidad, vivencias e intereses.

Pensar en una mediación para el aprendizaje en un contexto de educación formal, muchas veces resulta contradictorio. Es difícil intentar considerar otras dimensiones del tiempo, si la racionalidad que domina los currículos y las jornadas de clases, están en un tiempo cronológico. Sin embargo, creemos posible que la mediación emerja en el encuentro de un tiempo fractal (Briggs y Peats, 1999), el cual siempre será histórico en cada un-, pues no hay una regularidad, sino más bien una confluencia de multiplicidad de tiempos, como los ciclos propios y naturales. Por ello en un encuentro de mediación para el aprendizaje, el tiempo cobra relevancia para el aprendiente y mediador a partir del significado que cada uno le atribuye: es propio de quienes fluyen en este encuentro. Es un tiempo en el cual nos comprometemos plenamente con lo realizado, un tiempo creativo, donde cada experiencia se ofrece en un tiempo adecuado.

\section{8.- SÉPTIMA REFLEXIÓN: LA MEDIACIÓN COMO EXPERIENCIA VITAL EN CONTEXTOS DE INCERTIDUMBRES}

Como nos señala Morin (2014) vivir es afrontar sin cesar la incertidumbre, vivir es una aventura que conlleva en sí misma incertidumbres siempre renovadas, eventualmente con crisis o catástrofes personales o colectivas.

La educación no puede dejar fuera la experiencia vital que nos atraviesa cada día, las aulas deben estar vivas y no fosilizadas, permitir implicarnos mutuamente y promover experiencias situadas tanto personales, como colectivas. Entonces, desde la mediación pedagógica, las diversas problemáticas de la vida real son una oportunidad para experiencias de aprendizajes que posibilitan la autoorganización de los aprendientes, a partir de la emergencia de sus propias experiencias in situ.

En consecuencia, otorgamos lugar a la incertidumbre, propiciando una pedagogía de la pregunta, de la mano con Freire, comprendiendo que el educador no es quien tiene las respuestas, sino quien invita a problematizar según los intereses vitales, presentes en las aulas. Entonces, los encuentros de aprendizaje, se convierten en espirales abiertos con flujos múltiples, que permiten crear interrelaciones significativas que fluyen con el dinamismo cambiante de la vida. Se invita a que los acoplamientos estructurales surjan desde el caos, desde las conversaciones que adquieren sentidosignificado y que permiten la participación abierta de tod-s l-s interlocutor-s implicad-s.

Traer las dimensiones de la vida al aula y educar para la incertidumbre, resulta ser en estos tiempos un imperativo ético. Esta tarea requiere que quienes median estén en un estado de apertura, sensibilidad y flexibilidad, atento a sí mism-, como al clima del aula, de sus aprendientes, de los contextos personales, nacionales y 
mundiales que requieren ser traídos e incorporados en las aulas vivas. Un/a mediador/a con tacto pedagógico ${ }^{2}$ será sensible a lo sutil, a la incertidumbre que a veces se cierne por sobre todxs incluido su propio ser, entonces podrá navegar con mayor facilidad en los diversos mares y océanos, en las diversas geografías presentes cada día; para impregnarlas de sentido y profundidad en el autoaprendizaje de tod-s l-s aprendientes.

\section{9.- OCTAVA REFLEXIÓN: LA MEDIACIÓN COMO UNA RED DE CONVERSACIONES Y DIÁLOGOS ABIERTOS TRANSFORMADORES}

Como nos señala Maturana y Dávila (2015), lo humano se caracteriza por una manera de vivir que entrelaza nuestro lenguajear, sentires íntimos, emociones y haceres en redes de conversaciones que permean nuestras interacciones recursivas de coordinaciones consensuales, en el fluir del convivir. Entonces el conversar es un convivir siempre abierto a la posibilidad del surgimiento recursivo de nuevas coordinaciones consensuales que modulan el flujo de los cambios estructurales posibles, en un devenir de transformaciones del sentido de nuestro vivir-convivir abierto al infinito.

La mediación pedagógica puede ser entendida como una red de conversaciones que permite el flujo de interacciones entre todxs. Estar atentos a la cualidad de estas interacciones, desde una ética del encuentro que legitima a tod-s 1-s aprendientes es fundamental en todo proceso educativo dialógico. La mediación en el aula, debe promover la libre y diversa expresión de tod-s, posibilitando así, un educar para la ex-presión (Gutierrez, F. \& Prieto, D., 2002), vale decir, permitir exteriorizar, sacar fuera lo que unx tiene adentro, comunicar, manifestar, hacer público. El proceso educativo será tanto más rico, cuantas más posibilidades de ex-presión tengan 1-s aprendientes.

Bohm (1977), en su sugerente interpretación del sentido etimológico del diálogo, nos invita a concebir-

${ }^{2}$ Concepto desarrollado por Van Manen (1998)

48 Diez reflexiones sobre mediación pedagógica lo como una corriente de significado que fluye entre, dentro y a través de l-s implicad-s. Entonces el diálogo permite corrientes de significados al interior de un grupo humano, que posibilita el emerger de una nueva comprensión creativa, de algo que no se hallaba de modo alguno en el momento inicial, evidenciando así, su poder transformativo para cada uno de l-s implicads. Dialogar para Bohm (1977) no es discutir, ni debatir, sino la posibilidad de co-construcción de una corriente nueva de significados. En los diálogos auténticos, se requiere estar disponibles a cuestionar nuestra base de creencias fundamentales y todo lo que se encuentra tras ellas. El verdadero objetivo del diálogo para Bohm (1977), consiste en vincularse con el proceso del pensamiento personal, para posibilitar la transformación del proceso de pensamiento colectivo. Los diálogos auténticos resultan ser transformadores para tod-s l-s implicad-s, una de las búsquedas y tareas principales de una educación alternativa y de una mediación con sentido.

Sin diálogo en las aulas, no hay posibilidad de expresión libre, de pronunciar el mundo como dice Freire. La mediación pedagógica entendida como un diálogo abierto al interior de las aulas, resulta posible gracias a interacciones humanas horizontales, basadas en la confianza y en el respeto mutuo, "la confianza va haciendo que los sujetos dialógicos se vayan sintiendo cada vez más compañeros en su pronunciación del mundo". (Freire, 1988)

\section{0.- NOVENA REFLEXIÓN: LA MEDIACIÓN COMO INTEGRACIÓN DE SABERES}

La visión holística nos sitúa en la trama de la vida, entretejida por diversas perspectivas, comprendemos que todas las manifestaciones creadas son expresiones de un horizonte situado, es el resultado de un proceso cognitivo de seres humanos afectivos, sensibles, que piensan y crean en el seno de una cultura y en un territorio de intercambios. Los saberes son producciones 
colectivas en una totalidad fluyente en movimiento y que late en cada unx. (Najmanovich, 2016)

La ilusión de la superioridad epistémica, es el resultado de la colonialidad del poder, de una lógica imperialista (Grosfoguel, 2013) de una perspectiva que divide en una posicionalidad a la humanidad: la línea del ser y del no ser.

Reconocernos en una posicionalidad, que no es geográfica, sino política, nos lleva a una toma de conciencia sobre la diversidad epistémica y de la perversión que hay en la imposición de una sola matriz. La vivimos y la experimentamos en nuestro ser, en el vivir, en el acontecer de un despertar colectivo que ocurre en nuestro país $\left(\mathrm{Chile}^{3}\right)$. Podemos escuchar la polifonía de voces en un gran clamor, cuya consigna es la dignidad, la necesidad de reconocimiento, de mirarnos cara a cara, de vernos en la diversidad. Esta revuelta la vivimos en un emocionar que oscila entre la euforia y el miedo, ya que aquí se paga con la vida el alzamiento de la voz, la demanda por ser escuchado, la petición, pero también la exigencia por ser considerad-s. Mujeres, pueblos indígenas, activistas medioambientales, jóvenes, los invisibles, los sin nombre pujan por un espacio de consideración y sin embargo, la respuesta es la mutilación, la violencia, la deslegitimación.

Este acontecimiento marca en nuestra trayectoria un antes y un después, por ende, consideramos que la pedagogía debe tomarse en serio la diversidad epistémica.

En un encuentro para la mediación del aprendizaje, es necesario traer y considerar esta diversidad. Abrir espacios a esa pluralidad de formas de conocer la realidad, donde la mediación es un encuentro de epistemes. Por ejemplo, en la pedagogía de párvulos necesariamente hay un encuentro entre la episteme del adulto cuya racionalidad puede estar orientada a la productividad y los resultados, mientras que la episteme de l-s niñ-s fluye entre la intensidad del tiempo, en una aten-

${ }^{3}$ Nos referimos al contexto de la revuelta popular de octubre de 2019.

49 Diez reflexiones sobre mediación pedagógica ción permanente vivida en el presente; en la pedagogía popular el encuentro horizontal entre el conocimiento popular y el científico se entremezclan, no hay superioridad: se va construyendo a partir de las vivencias de quienes la conforman.

Por tanto, una mediación para el aprendizaje hemos de comprenderla como un encuentro en la cual no hay superioridad de epistemes sino una co-presencia radical.

\section{1.- DÉCIMA REFLEXIÓN: LA MEDIACIÓN COMO COMPROMISO POLÍTICO PARA EL EJERCICIO DE LA LIBERTAD}

Los patrones culturales de poder y dominación social como el patriarcado y la colonialidad del poder (Quijano, 2010), se han naturalizado y siguen vigentes en nuestra cultura, solo reproducen estructuras y relaciones jerárquicas y autoritarias, lo que produce identidades y clasificaciones, que definen lo que es incluido y lo que es excluido. Como consecuencia surge la violencia, los abusos, la discriminación, el sometimiento y la explotación.

El patriarcado como sistema en el cual se asienta nuestra cultura atribuye como principio absoluto lo masculino, identificado con el poder, la racionalidad, el orden, la agresividad; imponiendo el hombre su autoridad y apropiándose de la sexualidad de las mujeres, la reproducción y sus productos (Reguant, 2007). Este sistema inscrito y encarnado ha creado un orden simbólico que se reproduce en todos los ámbitos de la convivencia.

Deconstruir esta matriz en nuestro propio vivir y convivir, requiere de traer a la mano nuestra historia y biografía, reconociendo en ella el sello de estos patrones culturales en el cuerpo, en las emociones, en nuestras relaciones, sin dejar de ser un proceso que genera dolor. Reconocer, remirar, revivir, reflexionar, nombrar, escribir sobre la experiencia de dominación nos permite hacernos cargo de nuestras vivencias, pero a la vez nos sitúa en una posibilidad de resistencia desde los 
espacios más íntimos y cotidianos.

La mediación como una relación íntima, de proximidad, comprendida en el seno de la trama, necesita ser considerada como una relación basada en un vínculo que facilita la co-existencia, la co-evolución en una relación de respeto. No puede ni debe repetir patrones de dominación, por ello el mediador y mediado son parte de una coordinación de acciones, ocupan posiciones que van fluctuando: algunas veces se es mediado, en otras se media; hay una apertura, una ampliación, una implicación, una danza que permite transitar sin pretensiones de autoridad, ni supremacía, no hay imposición, hay una sugerencia, es una invitación.

\section{2.- REFLEXIONES FINALES}

Nuestra comprensión de la mediación educativa en el seno mismo de la vida, desdibuja las fronteras tradicionales desde las cuales se han entendido los procesos de enseñar-aprender, puesto que entrama a toda la humanidad como aprendientes, abriendo espacios a una pluralidad de formas y acercamientos al aprendizaje, involucrando coordinación de acciones respetuosas e implicadas entre los diversos actores, desde situaciones vitales, emergentes y caóticas. La mediación busca perturbar para abrir nuevos canales expresivos, invitar al diálogo abierto y creativo, producir transformaciones existenciales y dotar al aprendizaje de un sentido ético-estético-político para la vida. Entendemos la mediación como una provocación educativa.

La primera transformación para que esto sea posible implica un viaje sin retorno para tod-s l-s mediador-s, una transformación epistémica liberadora de las matrices jerárquicas que nos han conducido desde hace mucho en educación. Atrevernos a dejar atrás las lógicas lineales y las certezas controladoras en el aprendizaje.

Nuestro contexto actual de incertidumbre, nos interpela con fuerza a la transformación de nuestras praxis educativas. Bienvenid-s al viaje.

\section{Referencias bibliográficas}

Assmann, H. (2002). Placer y ternura en la educación. Madrid: Narcea.

Bohm, D. (1996). Sobre el diálogo. Barcelona: Kairos. Briggs, J. \& Peat. D. (1999) Las siete leyes del caos. Barcelona: Grijalbo.

Calvo, C (2012) Del mapa escolar al territorio educativo. La Serena: Ed. Universidad de La Serena.

Capra, F. (1992). El Punto Crucial. Buenos Aires: Troquel.

Freire, P. (1988). Pedagogía del oprimido. México: Siglo Veintiuno.

Gutiérrez, F. \& Prieto, D. (2002). Mediación Pedagógica, Apuntes para una educación a distancia alternativa. Programa EDUSAC: Guatemala

Hawking, S. (2018) La teoría del todo. Barcelona: Debate.

López de Maturana, S. (2017) Propensión a aprender, mediación y compromiso docente. Infancia, educación y aprendizaje, Vol. 3, $\mathrm{N}^{\circ} 1$

Maturana, H. y Dávila, X. (2015) El Árbol del Vivir. Santiago: MVP Editores.

Maturana, H. (2008) El sentido de lo humano. Santiago: JC Sáez Editor.

- (1990) Emociones y Lenguaje en Educación y Política. Buenos Aires: Paidós.

Maturana, H. y Varela, F. (2013) El árbol del conocimiento. Chile: Universitaria.

Morin, E (2014) Enseñar a Vivir. Buenos Aires: Nueva Visión.

Najmanovich, D. (2016) El mito de la objetividad. Buenos Aires: Biblos.

Prado, C. \& Gutiérrez, F. (s.f.) Siete claves para la Mediación Pedagógica. Serie Holografías No 3. Universidad La Salle: Costa Rica. 
Quijano, A. (2000) Colonialidad del poder y clasificación social. Journal of World-Systems research, VI, 2, Summer/Fall 2000, 342-386.

Reguant, D. (2007) Explicación breve del patriarcado. En: https://patagonialibertaria.files.wordpress. com/2014/12/sintesis-patriarcado-es.pdf

Van Manen, M. (1998) El tacto en la enseñanza. El significado de la sensibilidad pedagógica. Barcelona: Paidós.

Varela, F. (2016) El Fenómeno de la Vida. Santiago: JC Sáez Editor.

Galiza Ano Cero. 24/10/2013. Entrevista a Ramón Grosfoguel, URL: https://www.youtube.com/watch?v= 1oFM38D8ZEA\&feature=youtu.be 